\title{
Cultural epidemiology: An introduction and overview
}

\section{Citation}

Weiss, Mitchell G. 2001. "Cultural Epidemiology: An Introduction and Overview." Anthropology \& Medicine 8 (1) (April): 5-29. doi:10.1080/13648470120070980.

\section{Published Version}

doi:10.1080/13648470120070980

\section{Permanent link}

http://nrs.harvard.edu/urn-3:HUL.InstRepos:35642354

\section{Terms of Use}

This article was downloaded from Harvard University's DASH repository, and is made available under the terms and conditions applicable to Other Posted Material, as set forth at http:// nrs.harvard.edu/urn-3:HUL.InstRepos:dash.current.terms-of-use\#LAA

\section{Share Your Story}

The Harvard community has made this article openly available.

Please share how this access benefits you. Submit a story.

Accessibility 


\title{
Cultural Epidemiology: An Introduction and Overview
}

\author{
Mitchell G. Weiss \\ Department of Public Health and Epidemiology \\ Swiss Tropical Institute and University of Basel, Switzerland
}

Anthropology and Medicine

Special Issue on Cultural Epidemiology

In Press

May 2001

Correspondence to:

Dr. Mitchell Weiss

Professor and Head

Department of Public Health \& Epidemiology

Swiss Tropical Institute

Socinstrasse 57,

$\mathrm{CH}-4002$ Basel

Switzerland

E-mail: Mitchell-G.Weiss@unibas.ch 


\section{Abstract}

Although the value of interdisciplinary collaboration between epidemiology and anthropology is both widely acknowledged and hotly contested, effective international health policy and multicultural health programmes require it. The EMIC framework for cultural studies of illness was developed in response to such needs, and a cultural epidemiology emerged from that framework as an interdisciplinary field of research on locally valid representations of illness and their distributions in cultural context. These representations are specified by variables, descriptions, and narrative accounts of illness experience, its meaning, and associated illness behaviour. Specialised interactive qualitative and quantitative research methods provide a descriptive account, facilitate comparisons, and clarify the cultural basis of risk, course, and outcomes of practical significance for clinical practice and public health. This paper discusses the theoretical underpinnings of cultural epidemiology and an operational formulation for examining patterns of distress, perceived causes, and help seeking. Five additional papers in this special issue of Anthropology and Medicine indicate how the EMIC has been used and has engendered an ethnographically grounded cultural epidemiology. Although this overview and these papers are concerned exclusively with mental health, a complementary stream of active research on leprosy, tuberculosis, epilepsy, and other tropical infectious, neurological, and medical disorders is ongoing. Next steps for cultural epidemiology in mental health research are discussed, including (1) further clinicbased studies of psychiatric disorders, (2) studies of deliberate self-harm in clinic and community settings, (3) complementary cultural components of psychiatric epidemiological surveys, (4) baseline assessments to guide community programmes, and (5) contributions to intervention studies. 


\section{Cultural Epidemiology: An Introduction and Overview}

Differences in orientations and methods between anthropology and epidemiology hold the potential for both a complementary and antagonistic relationship. The power of quantitative methods applied to the study of the occurrence and determinants of diseases and disorders, as health professionals define these concepts, has made epidemiology a powerful tool for public health and clinical medicine. Ethnographic research and attention to local concepts of health and illness, as people in the general population understand and live with them, have stimulated developments in medical anthropology that contribute both to the agenda of academic anthropology and practical aims to inform health policy and clinical practice. ${ }^{1}$ The framework and methods of cultural epidemiology arose from efforts to achieve an effective interdisciplinary collaboration, finding appropriate and useful ways of integrating the frameworks and methods of anthropology and epidemiology.

At the outset our efforts concentrated on developing a framework and research instruments known collectively as the EMIC (Weiss 1997), and it was from experience in studies with these EMIC interviews that the concept of cultural epidemiology emerged. This paper discusses its conceptual and theoretical underpinnings, objectives and settings for which it is appropriate, and how to proceed. It also introduces several other papers in this special issue of Anthropology and Medicine that present key studies with the EMIC—-studies that have contributed substantially to cultural epidemiology.

A number of factors have stimulated this interdisciplinary collaboration. Accelerating social processes of migration, urbanization, and more frequent cross-cultural professional interactions in response to globalizing forces underscore the need for rapprochement and interdisciplinarity. Although globalization clearly has homogenizing effects, it may also 
accentuate a sense of individual ethnic identity within communities and the need to ensure that policy, planning, and practice are not only referenced to an international standard, but that they are also culturally sensitive and appropriate; in that regard, the reference to globalization in writing about development and health policy has been paired with localization in academic analysis, development policy documents, and the popular press (Junghans, 2001; World Bank, 2000; Schneider, 2001). Classical epidemiology provides a way to identify priorities and to evaluate the impact of policies and programmes, essential functions inasmuch as findings from such research influence priorities and the allocation of resources. Such information is not enough, however, to ensure that policy conforms to local needs, and for that a different but complementary set of questions and ways to answer them are needed, as we have suggested in an analysis of global and local approaches to health policy with reference to the example of mental health in India (Weiss et al., 2001a).

Although there is considerable overlap, higher-level institutions are typically more concerned with the epidemiological data that now regularly contribute to the calculation of DALYs (disability-adjusted life years), which have become the key indicator of the burden of disease in the statistics of WHO's annual World Health Report. Such data inform priorities and policy of global agencies based on comparisons across countries, and they guide national agencies based on comparisons within countries. Local agencies operating at ground level, where the success or failure of policy is ultimately determined, are more likely to be concerned with information about the specific features of the issues on their agenda and the communities they serve. Useful guidance requires consideration not only of the classical epidemiology of diseases and disorders, but attention also to the local experience of illness, its meaning, and both risk-related and help-seeking behaviours in the community. Transforming this agenda into an operational framework involve innovative integration of frameworks and both qualitative and quantitative research methods. The priority it places on 
clarifying both the nature and the distribution of illness experience, meaning, and behaviour make cultural epidemiology complementary to both medical anthropology and classical epidemiology.

\section{Scope of health research interests}

Our formulation of cultural epidemiology reflects the influence of developments in medical anthropology, psychiatric epidemiology, and efforts initially to integrate these two orientations in the framework for EMIC interviews. The first of these interviews was used to study leprosy and mental health in Mumbai (then Bombay), India, and it was influenced both by Kleinman's comprehensive representational formulation of the illness explanatory model (see below) and methods of psychiatric epidemiology, which were rapidly changing the assessment of psychiatric disorders in clinic and community studies and the character of clinical psychiatry. The EMIC relied more heavily on qualitative narrative data than did psychiatric epidemiological interviews, and as the name suggests, the EMIC was concerned with local concepts of illness, rather than professional concepts of disorder. Professional concepts of disorder were important, however, insofar as they specified particular health research interests and defined the clinical populations for clinic-based EMIC studies. The EMIC interviews also required a systematic and more complex approach to coding, analysis, and presentation of local categories of experience, meaning, and help seeking behaviour than the psychiatric assessments, which for the most part were concerned with the question of whether or not clinical problems fulfilled specific diagnostic criteria.

In addition to an EMIC interview, clinic-based studies of mental disorders used a diagnostic instrument (the Structured Clinical Interview for DSM-IIIR [SCID]) and a scale to assess the magnitude of depression (Hamilton Depression Rating Scale). This approach made 
it possible to relate local formulations of illness to professional assessments of disorder. In addition to a descriptive account of the patterns of distress, perceived causes, and the history and preferences for help seeking, the initial study of leprosy and mental health included an analytic component, examining how the meaning of leprosy for patients, as indicated by particular perceived causes, was related to the findings of clinically significant mental health problems. Perceived causes of leprosy emphasising fate and karma were associated with clinically significant depression, diagnosed with the SCID. The research also considered another important practical question about the role of culture, which was likely to be relevant for leprosy control, that is, adherence to treatment over the lengthy recommended course. Findings showed that specific perceived causes - namely, an imbalance of humours based on traditional Ayurvedic concepts (heat, cold, wind, bile, or phlegm)—predicted better adherence to a full recommended course of treatment.(Weiss et al., 1992).

The health problems that concerned this initial study included both a tropical infectious disease and questions of cultural psychiatry in clinical practice outside a EuroAmerican setting. These two streams have become an enduring focus of parallel interests for cultural epidemiology. Although this review and the selection of papers in this special issue are concerned with cultural psychiatry and mental health, research on infectious tropical diseases and other medical and neurological disorders also remains a priority. A pilot study of epilepsy in Vasai, a semirural area north of Mumbai, shortly after developing the first EMIC interview for leprosy and mental health, provided an initial opportunity to adapt the clinicbased approach to a field study. A recent, country-wide clinic and field study of leprosy in accessible areas of Sri Lanka — for which three parallel versions of the EMIC were prepared to study patients, nonaffected community residents, and health care providers — was undertaken to evaluate the impact of a social marketing campaign and to assist the country's leprosy eradication programme in its final phase. The leprosy programme-based research 
team developed vignettes supported by photographs as a focus for the interviews with nonaffected persons and health care providers, so that the investigators could compare the role of disease status and provider status on local cultural views of various types of leprosy. Findings identified achievements of the programme in promoting recognition, and shortcomings in changing attitudes and reducing stigma, and they also identified problems in the referral system, particularly among nongraduate Ayurvedic practitioners, that accounted for treatment delay.

Other infectious disease research has included a field-based study of childhood diarrheal disease in Central Thailand, completed by researchers in the Thai Ministry of Public Health. This work clarified cultural meanings of diarrhea and determinants of the use of oral rehydration by mothers for their children with diarrheal disorders in a rural area (Choprapawon, et al., 1991). After that, a WHO multicountry collaboration adapted the EMIC framework for research at six African sites studying onchocercal skin diseases. Findings showed the needs and feasibility of establishing a programme for the community-directed distribution of ivermectin, which is now promoted by the African Programme for Onchocerciasis Control (APOC) (Pan-African Study Group on Onchocercal Skin Disease, 1995; Akogun, et al., 2001). That group of studies also considered the distinctive gender aspects of the stigma affecting women with onchocercal skin disease (Vlassoff, et al., 2000).

Studies of tuberculosis in the Philippines (Auer, et al., 2000) and a comprehensive TB study of patients, nonaffected persons, and community-based health care providers in rural Pune District, India, have also been recently completed. A new multicountry study of gender and tuberculosis in Colombia, Malawi, India, and Bangladesh was initiated in December 2000 by the WHO Special Programme for Research and Training in Tropical Diseases. At a project implementation workshop the research group prepared an EMIC interview for a 
cultural epidemiological component of the study to answer questions about the role of gender (Uplekar, et al., 2001).

\section{Conceptual and theoretical underpinnings}

Despite many proponents of the priority and value of interdisciplinary collaboration, it is a controversial topic in both epidemiology and anthropology, and for some a highly contentious issue. An intentionally provocative editorial statement appearing in a recent issue of the International Journal of Epidemiology, published with five rejoinders, argued that the term social epidemiology is actually a misnomer. Instead of defining an interdisciplinary field of hybrid interests, "epidemiologists, sociologists and psychologists should stick to their field of scientific inquiry" (Zielhuis and Kiemeney, 2001). The prospect of cultural epidemiology, a less-known term, would clearly be equally offensive to these authors.

Some anthropologists have been no less critical of such interdisciplinary collaboration. Based on two years of participant observation in an epidemiology department in Spain, DiGiacomo (1999) argued that epidemiology and anthropology are not the natural allies they might appear to be. She found that it was difficult to maintain the priorities of anthropology under the influence of the "naturalistic epistemology of Western institutional medicine." Her critique discusses deficiencies of epidemiologists who would appear willing to collaborate, but who nevertheless fail to appreciate the web of relationships in their data and implications of the process by which they have acquired these data. She points out that they lack what Franzosi (1966) characterises as a theory of data, failing to appreciate the contexts in which it is embedded - considerations reminiscent of related discussions referring to a causal web (Dunn and Janes, 1986) and semantic illness networks (Good 1977). Her argument suggests that the problems result not just from the incompatibility of 
interdisciplinary concepts, but also from working with epidemiologists who require anthropologists to be more accommodating in a one-sided interdisciplinary collaboration.

Even those who would encourage it might question whether such interdisciplinary collaboration warrants designation of a special heading, cultural epidemiology. Does it not merely refer to the quantitative interests of medical anthropologists or the qualitative interests of epidemiologists? Why is such a new designation necessary? The rationale for defining a cultural epidemiology is justified by the fact that the priorities of both anthropology and epidemiology already constitute full agendas in well-established disciplines. This suggests that both fields may have insufficient motivation to expand their scope for working through integration of fundamental concepts and particular problems that arise in the course of forging such interdisciplinary collaboration. It might also be noted, as Richard Shweder pointed out in a recent paper explaining an analogous development_-"Why cultural psychology?"- that rather than working within the confines of an existing framework, the language and terminology that frames research may itself stimulate and shape innovation by articulating a fresh approach. He highlighted "the value of using language to symbolize "new beginnings"” (Shweder, 1999), in much the same way perhaps that coining the term new cross-cultural psychiatry was intended to encourage systematic attention to questions of cultural study that had been insufficient priorities of existing relevant disciplines (Kleinman 1977).

In the field of epidemiology, efforts to establish interdisciplinary links with the social sciences have focussed on sociology and social epidemiology (Berkman and Kawachi, 2000; Susser, et al., 1985). Anthropology has long-standing interests in development generally and particular interests in public health and medicine (Goodenough, 1963). Early efforts to link anthropology and public health, however, employed health-related ethnographic accounts of communities, which aimed to identify an authoritative community view, rather than explicitly considering the distribution of illness representations or other ethnographic features of 
communities. A volume of case studies, showing how communities reacted to health programmes, edited by Benjamin Paul (1955)—Health, Culture and Community—is a landmark contribution among such efforts to relate anthropology and public health. Two decades later as epidemiology became more influential, anthropological interests in public health acknowledged this development with the publication of another important edited volume, Anthropology and Epidemiology (Janes, et al., 1986). The work of Craig Janes (1999) has continued in that vein, and a recent study of his "explored the cultural epidemiology of rlung," a wind disorder among Tibetans living in Chinese Tibet. ${ }^{2}$

In elaborating the principles of an anthropological medicine Robert Hahn (1995), a thoughtful bridge builder, characterised the anthropological task as achieving "an understanding of the local framework. Anthropologists regard themselves as translators" ( $\mathrm{p}$. 104). He suggested that attention to this experience and meaning, listening and "understanding the patient's concepts of bodily (and mental and spiritual) function may also be critical to treatment" (p. 275). It is patients' perceptions that ultimately determine whether there is sickness, rather than a biomedical evaluation, which usually comes later (if at all). His consideration of anthropological medicine argues that the social and cultural environment not only shapes the nature of the illness, but it also plays a critical role in healing. Although professional criteria for disorder may guide treatment planning by clinicians and protocols for medical research, they do not determine patients' illness behaviour.

Hahn argued that anthropology and epidemiology share considerable common ground, even though "some anthropologists neglect the objective world or would wish it away," and "most epidemiologists would dispense with subjectivity entirely if they could" (p. 101), oblivious to the influence of the culture of their own discipline, which as Littlewood (2000) 
reminds us, is a powerful influence. The role of the medical anthropologist as a translator suggests that for ethnography to be useful, the anthropologist must be conversant with both local and professional concepts and categories. Similarly, to make use of epidemiological research, it is necessary to relate findings from studies of disease and disorder to suffering and context. Despite recognised differences in the priorities, frameworks, and methods of anthropologists and epidemiologists, to whatever extent both groups succeed in these endeavours, it follows, as Hahn argued:

Anthropologists have been doing epidemiology without knowing it, and epidemiologists have been anthropologists unawares... Although anthropologists and epidemiologists have been unwittingly and inevitably engaged in each other's disciplines, they may not have been doing so effectively. Practitioners of both disciplines may benefit from exploration of common roots and from explicit synthesis of methods (Hahn 1995, pp. 127-128).

Although he did not use the term cultural epidemiology, his argument indicated the need for it. As the following section shows, similar comments from an epidemiological vantage point have also made a case for interdisciplinarity.

\section{Culture, psychiatry, and psychiatric epidemiology}

Early in the 1970s when psychiatric epidemiology was sharply ascendant, Norman Sartorius, who headed the WHO Division of Mental Health, presented a paper on culture and the epidemiology of depression at a symposium of the Interdisciplinary Society of Biological Psychiatry in the Hague. He reviewed cultural differences in the clinical presentations of depression among different ethnic groups, and he suggested a priority for cultural research, stating that "the methodology of studies of the inter-relationship between culture and mental disorders needs to be further developed" (Sartorius, 1973, p. 484). Culture, he explained, was 
not merely a matter for exotic places. "Far too little is known about the major cultures of the world," and "depression should not be studied in isolation but seen as a condition that happens in its sociocultural environment" (p. 485). This view appeared to reflect a firm commitment for considering the role of culture in the psychiatric epidemiological studies that Sartorius was developing in his agenda for the WHO Division of Mental Health.

In the years that followed, however, as he and his colleagues proceeded with crossnational research on mental disorders, including the International Pilot Study of Schizophrenia (WHO, 1973) and the WHO study of Depressive Disorders in Different Cultures (WHO, 1983), these studies reflected a much narrower approach to cultural comparisons. They were limited for the most part to questions of whether rates of these disorders differed in different countries, rather than more fundamental consideration, as Sartorius himself had advocated, of the role of sociocultural contexts and the local cultural experience of these conditions, their meaning, and how these were related to behaviour.

Naturally, many anthropologists were disappointed, and joined by anthropologically oriented specialists in cultural psychiatry, they were highly critical of the methodological shortcomings and the failure of these WHO studies to take the mandate for cultural research seriously enough (Kleinman and Cohen 1997; Cohen 1992; Lin and Kleinman 1988). The example of WHO's approach to culture in these studies tacitly encouraged some researchers intrested in the comparative study of the rates of disorders in different cultures to use the term cultural epidemiology to describe their work (Yamamoto et al., 1998). That usage, however, which refers to comparative studies in classical psychiatric epidemiology, refers to a vastly different formulation of cultural epidemiology from the interdisciplinary approach that concerns us here.

In clinical practice, the focus on diagnosis that followed the introduction of criterionbased and axial diagnostic systems from the third edition of the American Psychiatric 
Association's Diagnostic and Statistical Manual (DSM-III) subsequently accommodated the inclusion of a so-called cultural formulation, introduced in DSM-IV. It provided the framework and guidelines for optional assessment of the cultural identity of the individual, cultural explanations of illness, attention to the psychosocial environment, and the relationship between patient and clinician. For those with a particular interest, the cultural formulation provided a framework for clinical assessment, teaching, and reporting of clinical case studies - all in a clinical context of the mainstream emphasis on diagnostic evaluation. Apart from the cultural formulation case studies published regularly in Culture, Medicine, and Psychiatry and sporadically in other journals, research to support the cultural formulation in clinical practice has been lacking. As findings from research in cultural epidemiology become available, however, they can be expected to support cultural assessment much as psychiatric epidemiology supports diagnostic assessment. The relationship of classical and cultural epidemiologies to their respective clinical assessments is analogous, and the relationship of psychiatric and cultural epidemiology is complementary (Figure 1).

\{Figure 1 about here\}

\section{Influential anthropological frameworks}

From the mid-1970s a focus on interpretive study of illness in medical anthropology began to concern itself with local cultural ways of characterising health problems as an alternative to the health professional orientations that typically guide clinical assessment. This was a challenging task, and considerable efforts were invested in finding suitable frameworks for distinguishing professional from local community orientations. Several of the frameworks that emerged from those efforts became highly influential and are now basic vocabulary in the field of medical anthropology. 
Even before that, Kenneth Pike had already adapted terminology from linguistics and linguistic anthropology in the mid-1950s (Headland, et al., 1990). He suggested the term emic to refer to the insiders' perspective, which provided an extension to social analysis of a phonemic approach to linguistic analysis (which is concerned with the basic unit of meaning in a particular language). Similarly, he suggested the term etic for the outsider's perspective, which provided an extension to social analysis of a phonetic approach to linguistic analysis; this referred to a professional formulation of the fundamental units of linguistic sounds (which can be represented symbolically and are independent of any particular language). Although this dichotomy has been used extensively in medical anthropology, it has also been applied more widely in other areas of anthropology and social analysis.

The distinction between disease and illness, introduced by Leon Eisenberg (1977), suggested a similar dichotomy but specific to medical anthropology. It distinguished community views based on local culturally influenced accounts of illness from health professional concepts of disease, based on formal considerations of structure, function, pathology, and pathophysiology. It also gave these terms disease and illness a distinctive technical meaning, which although widely appreciated as a guiding principle within medical anthropology would not necessarily be apparent to most people outside the field.

In the early 1980s Arthur Kleinman's explanatory model framework was fast becoming a major influence in medical anthropology. Initially he found the concept especially useful as he trained clinicians to interact empathically with patients in a manner that was sensitive and responsive to the cultural aspects of their clinical problems. To some extent, this training aimed to revise the clinical gaze through anthropological insights. Clinic- and fieldbased research studies to characterise explanatory models came later, and the particulars of these various research efforts varied considerably. 
The priority that Arthur Kleinman's formulation of explanatory models gave to illness experience, causal explanations, help seeking, and the approach to treatment influenced the development of the EMIC and the framework of cultural epidemiology. Nevertheless, as discussed elsewhere (Weiss, 2001), ambiguities in the usage of the term explanatory model have compromised its utility. Medical anthropologists have referred to explanatory models in three distinct ways: First, as Kleinman initially suggested, they specify a comprehensive account of the semantic networks linking experience and concepts of patients, healers, and all other concerned parties, and as such, they refer to perceived cause, onset, mechanisms, course, and the treatment of illness (Kleinman, 1980, p. 107). Second, the term is used in a narrower sense to refer more exclusively to the explanations, that is, the perceived causes of illness. It is in this sense that it is used in the section of the cultural formulation for clinical assessment in DSM-IV, elaborating “cultural explanations of the individual's illness" with reference to "the perceived causes or explanatory models that the individual and the reference group use to explain the illness" (APA [DSM-IV], p. 843-844). And third, it also refers to a structuralist model of the "cognitive distance" between patients and healers. Thus, the three usages refer to a comprehensive representational formulation, a focus on the explanatory, and a focus on the model.

The contribution of the first of these formulations made the explanatory model an extremely useful concept when it was first presented, and there were no other options nearly so attractive to integrate a cultural and clinical approach to practice and research. The development of more specific alternative concepts was facilitated in recent years, at least in part, by a drift towards the second explanatory formulation of the explanatory model. The third model formulation, which was also emphasised in Kleinman's (1980) influential treatise on the explanatory model framework, has ultimately had the least enduring appeal, even for Kleinman. In his reflections on the explanatory model published in 1995 he wrote: “Today, I 
am uncomfortable with the style and even the preoccupations of 'models', ethnocultural or other, which imply too much formalism, specificity, and authorial certainty, but models were definitely in my mind in the 1970s" (Kleinman, 1995, p. 7).

These three interpretive frameworks - emic-etic, disease-illness, and explanatory models - influenced considerable theoretical writing and research. As a theoretical base and stimulus to research, they helped to establish the field of medical anthropology as an academic subspecialty of anthropology and to facilitate collaboration between anthropology and both clinical medicine and public health. As a result of its ambiguities, however, the explanatory model framework has been largely supplanted by more specific concepts that provide a clearer focus for research. Littlewood and others have emphasised illness experience as an alternative interpretive framework: "If medical anthropology has its own impulse, it is to encourage understanding of patients' own illness experiences, the way in which they view their sickness and health, and the context in which such beliefs originate and continue" (Littlewood 1991, p. 1014).

Focussing on the explanatory and rational aspect of illness, Linda Hunt (1998) referred to causal explanations for an anthropological analysis of the way people - both patients and doctors - explain the cause of cancer. That term, however, may be confusing across disciplines because of another technical connotation; for epidemiologists causal explanations arise out of the professional process of causal inference from epidemiological data, rather than a local view of cause (Kaufman and Cooper, 1999). Byron Good's (1994) influential Morgan lectures emphasise the broader question of rationality as a cultural process, rather than cause as an individual cognitive psychological process. Similarly, Hunt and Mattingly (1998) refer to diverse rationalities of illness and healing. In Good's chapter in the above work on "illness representations in medical anthropology," he glosses the term 
illness representations as explanatory models ( $\mathrm{p} 42$ ), suggesting these are the substrate for illness attributions.

Medical pluralism and questions about cultural influences on illness behaviour, especially family-based and medical help seeking, and hierarchies of resort have long been important topics for anthropological research (Romanucci-Ross, 1969; Lewis, 1981). Cultural studies of risk-related behaviours, such as boiling drinking water, have also been subjects of classic anthropological contributions to public health (Wellin, 1955). Within public health, studies of illness behaviour guide health services policy and planning, especially the health belief model rooted in social psychology and David Mechanic's (1986) illness behaviour model, rooted in medical sociology. Good (1994, pp 41-45) critically reviews these and anthropological approaches to the study of illness behaviour.

Anthropological contributions apart from medical anthropology and its concerns with illness representations (experience, meaning, and behaviour) have also contributed substantially to our formulation of cultural epidemiology. Considering how anthropology explains culture, Dan Sperber argued that cultural study may be regarded as study in human populations of the distributions of representations, and his theory refers to epidemiologies of representations (Sperber 1994, 1985) and beliefs (Sperber, 1990). He calls it an epidemiology because he is concerned with the "distributions of representations (and consequently the modifications of the environment which are causally involved in these distributions)" (Sperber, 1985, pp. 86-87). He suggested that the epidemiology of diseases serves as a paradigm for examining the distributions of representations and beliefs, which include folk concepts, religious beliefs, oral narratives, and so forth. Leaning heavily on cognitive psychology in his theory of culture, Sperber explained, "Just as an epidemiology of diseases has to be rooted in individual pathology, an epidemiology of representations has to be rooted in cognitive psychology" (Sperber, 1985). Epidemiologists are concerned not only with a 
descriptive account of diseases, but also analytic study of their determinants. Similarly, Sperber's metaphor acknowledged both a descriptive and an analytic epidemiology. He asked why some ideas proliferate, trying to "explain why some representations are more successful - more contagious - than others" (Sperber, 1994, p. 54).

\section{Objectives and methods of cultural epidemiology}

In contrast with Sperber's epidemiology of beliefs and cultural representations of various kinds, our cultural epidemiology deals with local representations of illness. The tasks of identifying and analysing valid representations and determining how they are distributed are interdependent. Illness representations are specified by variables, descriptions, and narratives accounting for the experience of illness, its meaning, and illness-related behaviours that affect risk or reflect help seeking. To understand what they are and how they operate, carefully integrated qualitative and quantitative research is required. Ethnographic study is well-suited to identify valid representations with reference to the framework of locally valid categories of experience, meaning, and behaviour. Semi-structured EMIC interviews may then account for the distribution of these illness representations, making use of narratives to clarify the categories and to explain their role and significance.

The first phase of cultural epidemiological research requires careful attention to the context and concepts of illness. Without that an EMIC interview cannot be emic. In the clinic this means cultivation of an ethnographic gaze to complement the standard clinical approach; in the community, this may involve explicit methods of ethnographic research. Researchers have developed this component of the research in different ways. The reports in this issue of the two studies led by Raguram and Jadhav show how they each approached the ethnographic tasks in their clinic-based studies in Bangalore and London. Ruth Lee also relied not just on 
$-19-$

extensive clinical experience and activities in a hospital-based multicultural health service, but also extensive involvement with community agencies and services, and ethnographic study through this network. The paper by Chowdhury focuses exclusively on an explicit ethnographic phase of the research in three villages at each of two field sites in the Sundarban Delta of West Bengal. Experience from this phase of the work informed construction of EMIC interviews for the second phase of research.

\section{Structure of EMIC interviews and approach to analysis}

The structure of EMIC interviews focusses on illness experience, its meaning, and related behaviour, which have been made operational as patterns of distress (PD), perceived causes (PC), and help seeking (HS) (Figure 2). Initially referring to the comprehensive representational formulation of the explanatory model, EMIC was originally conceived as an acronym for the Explanatory Model Interview Catalogue, although the above discussion of anthropological frameworks indicates that the reference to explanatory model in the earlier formulation has now become ambiguous. Because the EMIC framework and EMIC interviews aimed to be emic, insofar as possible, they inevitably required local adaptation to ensure they were appropriate in the local cultural context, for the particular health problems under consideration, and for the scope of particular research interests. Unlike instruments developed for psychiatric epidemiology, the intention was not to create a single EMIC interview, but rather a framework that would guide locally relevant research with reference to locally specified research objectives.

\{Figure 2 about here\} 
Some aspects of the process of developing EMIC interviews resemble considerations for psychiatric epidemiological instruments. Interrater reliability is important for both and has been studied (Chowdhury, et al., 2001a). Also like classical epidemiology, objectives of cultural epidemiological research may be descriptive, comparative, or analytic. In diverse cultural settings a descriptive account clarifies distinctive features of the clinic or community and the relevance and limitations of textbook accounts of clinical disorders. Comparative aims may focus on the relationship of illness experience, meaning, and behaviour in different nations, regions, clinics and practice settings, or before and after implementing policy or establishing programmes. Analytic aims consider the impact of particular features of distress, perceived causes, or prior help seeking on practical clinical or public health outcomes. For example, findings from the initial EMIC study of leprosy and mental health, noted above, served such analytic interests.

A noted at the outset, the development and structure of the EMIC have been discussed in a previous paper (Weiss, 1997). Briefly put, each section (PD, PC, and HS) contains introductions communicating the intent of the interview, which emphasise the priority of a respondent's ideas rather than anticipation of what it might seem the interviewer would judge to be correct. The format of questions in each section includes open-ended queries in the first phase of inquiry to identify what immediately comes to mind without prompting for specific response categories. Basic questions of interest include "What is the problem?" "What is the cause?" and "What kind of help did you get?" The next phase of inquiry in each section probes various locally relevant categories that had not been mentioned. Summary questions in the third phase of inquiry further specify the relative significance and overall prominence of the various categories of illness experience, meaning, and behaviour.

The interview typically distinguishes whether a response category is reported in the answers to open-ended queries or probed queries in each section; and this distinction is 
usually indicated by presenting frequencies of those categories reported spontaneously without prompting and frequencies for categories identified only after prompting (referred to as probed or screened in some reports). For example, if a respondent explained, "My problem resulted from quarrels with my boss at work," it would be coded spontaneous if this account came without prompting in response to the question, "What is the cause of the problem?" If the same remark came in response to a prompted question- "Was this a result of problems at work?"- then it would be coded as a prompted response. The distinction between spontaneous and prompted responses identifies those representations of illness that are relevant, but which may not be readily apparent without an explicit query. Experience from EMIC interviews shows that summary queries (eg, most troubling symptom, most important perceived cause, first help seeking, etc.) are not necessarily included among the spontaneous responses and may require prompting.

The interview forms are structured to provide instructions to the interviewer, introductions to each section, questions, and space for data codes and narratives; the format facilitates the process of interviewing, coding responses, and managing categorical and prose data. The prominence of any category is computed from how it is reported in the course of the interview. Responses that are reported spontaneously, emphasised, and affirmed in response to the summary questions have the highest prominence, and those that are not reported at all have zero prominence. These prominence rankings may provide a more sensitive indication of the role of the various categories of distress, perceived causes, and help seeking, and facilitate correlational analysis. They also facilitate analysis of hypothesised relationships between aspects of experience, meaning, or behaviour with other aspects of illness, such as diagnosis, severity, stigma, and outcomes of practical clinical or public health significance (eg, treatment delay, adherence to lengthy course of treatment, and return for recommended follow-up). 
The number of coded categories of PD, PC, and HS may be large in some studies to include relevant local categories of interest. Data reduction may therefore be useful to group these interview response variables in the course of analysis. For example, marital problems, other family quarrels, and problems with people at work may be summarised under a grouped category of social or interpersonal problems to permit examination of this broader concept, rather than the individual category variables, which also remain available for consideration, as required. Data management features of statistical or spreadsheet programs are used to reconfigure these analysis variables from interview responses. The overall approach to the analysis of EMIC interviews is indicated in Figure 3.

\section{\{Figure 3 about here\}}

\section{Stigma}

Stigma is a disturbing social component of many chronic illnesses that has become an important topic for public health generally, and especially for mental health. Illness-related stigma - characterised by medically unjustifiable avoidance, or by disgrace and ignominy resulting from the social response to the illness — contributes additional suffering beyond the symptoms. Efforts to avoid this social component of illness may also delay or obstruct appropriate help seeking and the willingness to continue treatment over the recommended course (Sirey, et al., 2001). WHO's Nations for Mental Health Programme (1997) regards stigma as part of the so-called hidden burden of mental illness, and reducing the stigma of mental illness has become a prominent feature of population-based mental health policy. Insofar as illness-related stigma may be considered in large measure a social and cultural phenomenon, the manifestations of stigma and the conditions that are stigmatised may vary across cultures. Indicators for assessing stigma therefore need to be considered and validated 
locally. That is just the kind of problem, however, that cross-cultural research has struggled with in the past, able to offer little more than what Bruce Dohrenwend described as his "Rube Goldberg" solution for accommodating cultural interests in psychiatric research (Dohrenwend and Dohrenwend, 1974).

Cultural epidemiology and the EMIC give special attention to assessing stigma as an elaboration of patterns of distress, and locally coherent concepts of stigma have been evaluated in several studies by statistically demonstrating the internal consistency of indicators with reference to the Cronbach's alpha statistic (Cronbach, 1951). These indicators and a scale based on their combined effect provide a means of assessing and comparing stigma for different disorders, in different settings, and over time. With the emphasis on stigma reduction in public health, this contribution of cultural epidemiology, as described in the the paper comparing the stigma of depression among outpatients in Bangalore and London in this issue, provides an approach to locally validate and assess self-perceived stigma among patients in clinics (Weiss, et al., 2001b).

Assessment of stigma has also been a feature of recent field studies of nonaffected persons and health care providers with reference to vignettes depicting problems of interest (Chowdhury, et al., 2000). Comparing stigma among groups defined by whether they are affected with a particular health problem, the stigma of that condition is typically reported as greater among nonaffected respondents with reference to a vignette than self-reported stigma among people suffering from that condition (Pan-African Study Group on Onchocercal Skin Disease, 1995; consistent also with unpublished data from clinic and field studies of leprosy in Sri Lanka). Such locally validated scales that quantify the magnitude of stigma and narrative accounts that specify the nature of stigma should be useful to guide and evaluate community interventions for reducing stigma. 


\section{Qualitative data}

Narrative detail and notes elaborating the interview process are recorded and maintained as an integral component of the data set in a prose database. Such qualitative data are prepared either from the notes of a second person working with the interviewer, or from a tape recording that is transcribed verbatim or used as a reference to help the interviewer complete the data record. These qualitative data from the interviews are analysed with reference to the structure of the interview and with reference to coded categorical data. Descriptive frequencies without such prose elaboration to clarify what the categories represent are difficult to interpret and less useful.

For example, in the analysis of perceived causes of leprosy from the first study with an EMIC interview, noted above, nearly $30 \%$ of patients identified food as the cause of their condition. The ways in which food was understood to have brought it about, however, varied considerably. For some, it was the result of food having been contaminated, for others it was because of eating religiously proscribed foods, and for still others it was the result of a culturally inappropriate mixing of foods, such as fish and milk (Weiss, et al., 1992). Despite the common element, appropriately coded as foods, each of these accounts reflected a distinct set of concerns and associations that explained how food was thought to be a cause.

The interrelationship of quantitative and qualitative data is a priority for cultural epidemiology, and approaches to their interactive analysis have been developed. Analytic approaches in classical epidemiology are typically concerned with identifying relationships between risk factors and disease outcomes, or statistically valid differences in the impact of alternative interventions on course and outcome. Demonstrating the existence of such relationships is often considered the objective of research and an endpoint for analysis. With the availability and ready access to a rich qualitative data, however, the identification of a relationship raises additional relevant questions, beyond specifying the correlation, about the 
nature and dynamics of that relationship. After identifying an association from quantitative data, qualitative data help to explain what it means.

The study of stigma reported by Raguram et al. (1996), for example, showed a positive relationship between stigma and the magnitude of depression, as measured by computing a stigma scale and correlational analysis with ratings on the Hamilton Depression scale. By selecting illustrative subjects with prominent depression and high self-perceived stigma, and examining their narrative accounts of stigma and depression, we learn how these respondents account for stigma and depression. Other aspects of the narrative account of these particular respondents may also be examined. By selecting the exceptional respondents off the curve (ie, high depression and low stigma or low depression and high stigma), we may also learn why stigma is not a problem for some people for whom we expect it to be because of their severe depression, and how it may unexpectedly be a problem (based on the relationship in the group) for people with a lesser degree of depression. This approach may be especially useful for answering practical clinical and programmatic questions qualitatively, such as vulnerability to stigma and effective coping with depression.

Software and methods for computer-assisted analysis of qualitative data have improved greatly over the past decade, making it easier to access specific coded segments from selected records, to recontextualise these segments, and to compare them with other records selected according to complex criteria. Analytic software facilitates the interactive approach to quantitative and qualitative analysis, making it possible to proceed systematically with moderate-size or larger data sets. Features that readily enable structured coding of text segments with reference to responses from a semi-structured interview, and the ability to import quantitative data as selection criteria from the database used for quantitative analysis make it possible for cultural epidemiology to avoid the pitfalls of simplistic reductionism and 
to make appropriate use of the combined power of qualitative and quantitative research methods.

\section{Introduction to the papers in this issue}

The group of studies presented in the following papers in this issue of Anthropology and Medicine have made important contributions to the development of cultural epidemiology for mental health. Each of them proceeded in the context of collaboration and as a part of a cultural research network, and all of the collaborators have been keenly aware of and motivated by acknowledged needs for better tools and methods for cultural research (Rogler, 1989; Marsella and White, 1982; Fabrega, 1974). Depression has been an especially important topic, not only because of its importance in clinical practice and public health, but also because of the ways that culture shapes the complex interactions of biomedical, psychological, and social aspects of depression as distress and disorder.

The research reported by Raguram et al. (2001) at the National Institute of Mental Health and Neurosciences (NIMHANS) in Bangalore was among the first studies of depression with the EMIC. It was motivated by recognition of problems in clinical practice in India that arose from exclusive attention to the formal professional (etic) concepts of depressive disorder; these were repeatedly found to be inadequate guides to the culturally appropriate treatment of the various clinical problems that arose under the heading of depression. Research with the EMIC and collaboration at NIMHANS has informed practice and teaching and contributed substantially to the conceptual framework and methods of cultural epidemiology. The paper indicates how an EMIC interview for depression was developed in Bangalore, examines quantitative and qualitative findings, and discusses the implications for culturally relevant approaches to treatment. 
From his experience and contributions to the development of the research at NIMHANS and the study of Raguram, Jadhav used the Bangalore version of the EMIC to develop a cultural study of depression in London, England (Jadhav, et al., 2001). As he proceeded to develop a British EMIC interview for depression, he carefully considered historical features of depression in Europe (Jadhav, 2000) and ethnographic considerations from a variety of sources. By starting with an instrument developed initially from experience in India, this process of adaptation reversed the usual direction of the migration of professional research methodologies. The diversity and multiplicity of illness experience, meaning, and behaviour that he found from study of white Britons indicates the value of a cultural approach not only for Asian, African, and Latino cultures, but for the majority populations of Euro-American societies as well, as Sartorius (1974) had recommended nearly three decades ago. The discussion also compares findings from the studies in Bangalore and London.

Another aspect of the comparative analysis is presented in the study of stigma that follows, which describes the approach to locally validating stigma scales and assessment of stigma. Weiss and colleagues (2001b) use data from the studies reported in the two previous papers to compare the nature of stigma and its prominence in the clinical samples at both sites. Considering an assessment of stigma with reference to locally validated scales and accounts of specific features of stigma, this paper demonstrates the complementarity of quantitative and qualitative approaches for cross-cultural comparisons and show how to proceed. Beginning with a critical discussion of the concept of stigma that recognises the ambiguity and complexity of the term, and with its consideration of the pitfalls of a simplistic overinclusive formulation, the paper raises important questions for broader critical discussion of the meaning of stigma and how it may appropriately apply to pertinent questions for public health. 
The research reported by Ruth Lee and colleagues (2001) focusses on the qualitative data from an EMIC study of neurasthenia and chronic fatigue among Chinese immigrants in Toronto, Canada (Lee, et al., 2001). This research was conducted in cooperation with a group of investigators studying neurasthenia and chronic fatigue syndrome in Los Angeles, Hong Kong, Taiwan, mainland China, and India (Lin, et al., 2001; Lee et al., 2000). Ruth Lee's report emphasises the influence of the migration experience and the role of social stressors on this widespread clinical problem; the condition itself is a matter of particular interest because of the distinctive cultural meanings of similar patterns of distress among the Chinese immigrant and the North American populations. The Chinese patients in her study were largely dissatisfied with the inability of Canadian doctors they had consulted to appreciate the nature of their problems, but practitioners of traditional Chinese medicine they preferred were often too costly to use. Her analysis describes an approach to research responsive to the challenges confronting health systems that serve multicultural populations, and it indicates how baseline data may inform the operations of such a programme.

Chowdhury's efforts to develop a mental health programme linked with primary health services in the underserved rural Sundarban Delta of West Bengal, India, extend the clinical focus of the prior studies to field research for community mental health (Chowdhury, et al., 2001b). This report reviews development of the project and presents the first phase of ethnographic study that informed development of a mental health programme and provided the information needed to construct locally valid EMIC instruments to study patients, community residents who were not personally affected by overt mental illness themselves or in their immediate family, and health care providers who comprise the extended local health system. These practitioners comprised a wide range of provider types apart from the few doctors in government services and accredited private practitioners. Based on participatory and traditional ethnographic methods, the paper indicates how the researchers identified local 
categories of illness experience, meaning, and behaviour for inclusion in the EMIC

interviews. Field experience also informed the composition of vignettes that were used as the focus of inquiry for interviews in the community with nonaffected persons and health care providers.

Community-based field studies are becoming an increasingly important application of cultural epidemiological research. Dr. S.R. Parkar is conducting a comparable ethnographic and cultural epidemiological study in an urban slum in Mumbai with special attention to gender and women's mental health. These studies are especially well suited to identify local priority issues and problems, and they also indicate how the existing health system deals with them. Community-based programmes need such information to inform policy for health promotion and illness prevention, and findings from these studies are already guiding community mental health services at the rural and urban study sites on Sagar Island in the Sundarban region and in Mumbai (Weiss, et al., 2001a).

\section{Next steps for cultural epidemiology}

Experience from these and other studies and from consideration of the background and concepts presented in this overview suggest five areas for continuing and new mental health research in cultural epidemiology. Although distinctive considerations recommend each of them, their anticipated contributions also overlap:

1. Continuing studies of the clinical cultural epidemiology of psychiatric disorders, such as depression, schizophrenia, and other severe and common mental disorders in specialty and general health service settings.

Experience with the EMIC began with clinic-based studies, and they should continue. Research in cultural epidemiology should support clinical practice by providing local data to 
aid interpretation of explicit cultural assessments, such as the cultural formulation of DSM-IV. Research on a larger scale should also aid in further developing cultural formulations, as anticipated for the next editions of the two international diagnostic systems - WHO's International Classification of Diseases (ICD) and the American Psychiatric Association's Diagnostic and Statistical Manual (DSM).

2. Studying deliberate self-harm in clinical and community settings.

Deliberate self-harm is an especially important topic for cultural research because of the complex mix of factors that lead to it and the role of associated problems that are not necessarily congruent with mental disorders. Reducing suicides and providing appropriate treatment for patients after episodes of deliberate self-harm require sensitivity to the social and cultural meanings of these behaviours and their determinants. Even though these events do not define mental disorders, they are nevertheless an indication of serious emotional distress and a priority for mental health policy.

3. Incorporating complementary cultural epidemiological components of psychiatric epidemiological surveys.

Psychiatric epidemiology has provided data that suggest the immensity of the burden of mental health problems, and a recent editorial by Rachel Jenkins (2001) discusses various ways that psychiatric epidemiology should inform mental health policy. Documenting the burden of disease and disorder, however, is only one step on a path towards effective planning; it also requires a process responsive to relevant local concepts, needs, and priorities. Introducing a cultural epidemiological component in psychiatric epidemiological surveys will provide complementary information about experience, meaning, and behaviour for the disorders, which are now the exclusive focus for mental health policy.

4. Incorporating cultural epidemiological assessments in baseline surveys for community programmes. 
In addition to cultural research to support clinical assessment and the information that larger surveys provide, community-based research is needed to support the specific agendas of local mental health programmes. Priority issues include severe mental disorders, common mental disorders in primary health care, deliberate self-harm, and other locally defined mental health problems and concerns that may not be covered by the ICD or DSM lists. Local programmes require guidance from cultural epidemiological research to ensure planning takes into account relevant features of the community, and the composition and mental healthrelated activities of the extended health system already in place.

\section{Contributing to intervention studies.}

Incorporating a cultural epidemiological component of clinical and community-based intervention studies would clarify the relationship between perceived and observed effects. It would also help to explain associations by using methods discussed in this paper for linking quantitative and qualitative methods.

Advances in the interdisciplinary research capacity of anthropology and epidemiology now offer opportunities for addressing cultural dimensions of health for which, until recently, needs were clear but methods lacking. We should, and we can, set a more ambitious research agenda on which cultural considerations are no longer just an attractive-but-unavailable option. In view of the needs of a world struggling to accommodate the homogenizing forces of globalization and the localizing needs of distinctive communities and cultures, the capacities of cultural epidemiology need to be developed and used.

\section{Acknowledgement}

Proceedings of the Workshop on Cultural Epidemiology and Experience with the EMIC, held at the Swiss Tropical Institute, 21-25 February 2000, contributed to the 
$-32-$

development of this paper. Support from the Swiss National Science Foundation, Grant \#32-51068.97, Cultural Research for Mental Health, is gratefully acknowledged.

Development of the first EMIC interview, used for study of leprosy and mental health, as discussed in this paper, and collaboration with the Department of Psychiatry, KEM Hospital, Bombay, then headed by Dr. D.R. Doongaji, Honorary Professor, was supported by an NIMH Research Scientist Development Award (RSDA) MH00616 and a fellowship of the American Institute of Indian Studies. I am especially grateful to Dr. Sushrut Jadhav for his contributions to the development of the EMIC framework, his substantive and organizational contributions to this special issue of the journal, and his helpful comments on an earlier version of this paper. 


\section{Reference List}

Akogun, O. B., Audu, Z., Weiss, M. G., Adelakun, A. O., Akoh, J. I., Akogun, M. K., Remme, H., Kale, O. O. 2001. Community-directed treatment of onchocerciasis with ivermectin in Takum, Nigeria. Tropical Medicine and International Health, 6, 232243.

Auer, C., Sarol, J., Tanner, M., Weiss, M. G. 2000. Health seeking and perceived causes of tuberculosis among patients in Manila, Philippines. Tropical Medicine and International Health, 5, 648-656.

Berkman, L. F., Kawachi, I. 2000. A historical framework for social epidemiology. In: Berkman, L. F., Kawachi, I. (Eds.). Social Epidemiology. New York: Oxford University Press.

Choprapawon, C., Chunsutiwat, S., Kachondham, Y. Weiss, M. G. 1991. Cultural study of diarrheal illness in two villages of Central Thailand. Journal of Diarrhoeal Disease Research, 9, 204-212.

Chowdhury, A. N., Sanyal, D., Bhattacharya, A., Dutta, S.K., Banerjee, S., De, R., Bhattacharya, K., Palit, S., Bhattacharya, P., Mondal, R. K., and Weiss, M. G. 2001a. Interrater reliability of the EMIC in a pilot field study in West Bengal. International Medical Journal, 8, 25-29.

Chowdhury, A. N., Chakraborty, A.K., Weiss, M. G. 2001b. Community mental health and concepts of mental illness in the Sundarban Delta. Anthropology and Medicine, 8, ????.

Chowdhury, A. N., Sanyal, D., Dutta, S. K., Banerjee, S., De, R., Bhattacharya, K., Palit, S., Bhattacharya, P., Mondal, R. K., and Weiss, M. G. 2000. Stigma and mental illness: 
pilot study of laypersons and health care providers with the EMIC in rural West Bengal, India. International Medical Journal, 7, 257-260.

Cohen, A. 1992. Prognosis for schizophrenia in the Third World: a re-evaluation of crosscultural research. Culture, Medicine, and Psychiatry, 16, 53-75.

Cronbach, L. J. 1951. Coefficient alpha and the internal structure of tests. Psychometrica, 16, 297-334.

DiGiacomo, S. M. Can there be a "cultural epidemiology?" Medical Anthropology Quarterly, $13,436-457$.

Dohrenwend, B. P., Dohrenwend, B.S. 1974. Social and cultural influences on psychopathology. Annual Review of Psychology, 25:417-452.

Dunn, F. L., Janes, C. R. 1986. Introduction: medical anthropology and epidemiology. In: Janes, Craig R., Stall, Ron, Gifford, Sandra M. (eds.). Anthropology and Epidemiology. Dordrecht: D. Reidel, Pp. 3-34.

Eisenberg, L. 1977. Disesae and illness: distinctions beween professional and popular ideas of sickness. Culture, Medicine, and Psychiatry, 1, 9-23.

Fabrega, H. 1974. Problems implicit in cultural and social study of depression. Psychosomatic Medicine, 36, 377-398.

Franzosi, R. 1996. A sociologist meets history: critical reflections upon practice. Journal of Historical Sociology, 9, 354-392.

Good, B. J. 1994. Medicine, Rationality, and Experience: An Anthropological Perspective. Cambridge, UK: Cambridge University Press.

Good, B. J. 1977. The heart of what's the matter. The semantics of illness in Iran. Culture, Medicine, and Psychiatry, 1, 25-58.

Goodenough, W. 1963. Cooperation in Change: An Anthropological Approach to Community Development. New York: Russell Sage Foundation. 
Government of India. 1999. Accidental Deaths and Suicides in India, 1997. National Crime Records Bureau, Ministry of Home Affairs. New Delhi: Government of India.

Hahn, R. A. 1995. Sickness and Healing: An Anthropological Perspective. New Haven: Yale University Press.

Headland, T. N., Pike, K. L., Harris, M. (Eds.) 1990. Emics and etics: The insider/outsider debate. Frontiers of Anthropology Series, No. 7. Newbury Park and London: Sage.

Hunt, L. 1998. Moral reasoning and the meaning of cancer: causal explanations of oncologists and patients in Southern Mexico. Medical Anthropology Quarterly, 12, 298-318.

Hunt, L., Mattingly, C. 1998. Introduction: diverse rationalities and multiple realities in illness and healing. Medical Anthropology Quarterly, 12, 267-272.

Jadhav, S., Weiss, M. G., Littlewood, R. 2001. Cultural experience of depression among White Britons in London. Anthropology and Medicine, 8, ??-??.

Jadhav, S. 2000. The cultural construction of Western depression. In: Skultans, V., Cox, J. (Eds.). Anthropological Approaches to Psychological Medicine: Crossing Bridges. London: Jessica Kingsley Publishers, Pp. 41-65.

Janes, C. R. 1999. Imagined lives, suffering, and the work of culture: the embodied discourses of conflict in modern Tibet. Medical Anthropology Quarterly, 13, 391-412.

Janes, C. R., Stall, R., Gifford, S. M. (Eds.). 1986. Anthropology and Epidemiology: Interdisciplinary Approaches to the Study of Health and Disease. Dordrecht: D. Reidel Publishing Company.

Jenkins, R. 2001. Making psychiatric epidemiology useful: the contribution of epidemiology to government policy. Acta Psychiatr Scand, 103, 2-14.

Junghans, T. 2001. Editorial: Global - national - local. Tropical Medicine and International Health, 6, 1-3. 
Kaufman, J. S., Cooper, R. S. 1999. Seeking causal explanations in social epidemiology. American Journal of Epidemiology, 150, 121-126.

Kleinman, A. 1995. Writing at the Margin: Discourse between Anthropology and Medicine. Berkeley: University of California Press.

Kleinman, A., Cohen, A. 1997. Psychiatry's global challenge. Scientific American, 276, 7477.

Kleinman, A. 1980. Patients and Healers in the Context of Culture. Berkeley: University of California Press.

Kleinman, A. 1977. Depression, somatization and the "new cross-cultural psychiatry." Social Science and Medicine, 11, 3-10.

Krishnakumar, A. 2001. Weavers in distress (cover story). Frontline 18, 5-21 (2001 April 27).

Lee, R., Rodin, G., Devins, G., Weiss, M. G. 2001. Illness experience, meaning, and help seeking among Chinese immigrants in Canada with chronic fatigue and weakness. Anthropology and Medicine, 8, ??-??.

Lee, S., Yu, H., Wing, Y., Chan, C., Lee, A. M., Lee, D. T. S., Chen, C. N., Lin, K. M., Weiss, M. G. 2000. Psychiatric morbidity and illness experience of primary care patients with chronic fatigue in Hong Kong. Am J Psychiatry, 157, 380-384.

Lewis, G. 1981. Cultural influences on illness behaviour: a medical anthropological approach. In: Eisenberg, L., Kleinman, A. (Eds.). The Relevance of Soical Scince for Medicine. Dordrecht: Reidel, Chapter 7, Pp. 151-162.

Lin, K. M., Lin, M., Zheng, Y. 2001. Neurasthenia and chronic fatigue syndrome: lessons from cross-cultural study. In: Yilmaz, A. T., Weiss, M. G., Riecher-Rössler, A. (Eds.). Cultural Psychiatry: Euro-International Pespectives. Basel: Karger, Bibliographica Psychiatrica Series, No. 169, Pp. 68-80. 
Lin, K. M., Kleinman, A. M. 1988. Psychopathology and clinical course of schizophrenia: a cross-cultural sperspective. Schizophrenia Bulletin, 14, 555-567.

Littlewood, R. 2000. Psychiatry's culture. In: Skultans, V., Cox, J. (Eds.). Anthropological Approaches to Psychological Medicine: Crossing Bridges. London: Jessica Kingsley Publishers, Pp 66-93.

Littlewood, R. 1991. From disease to illness and back again. Lancet, 337, 1013-1016.

Marsella, a. and White, B. (Eds.). 1982. Cultural Conceptions of Mental Health and Therapy. Dordrecht: Reidel.

Mattingly, C. 1998. In search of the good: narrative reasoning in clinical practice. Med Anthropology Quarterly, 12, 273-297.

Mechanic, D. 1986. Illness behavior: an overview. In: McHugh, S., Vallis, T.M. (Eds.). Illness Behavior: A Multidisciplinary Model. New York: Plenum Press.

Menon, Parvathi. 2001. A farm crisis and suicides (cover story). Frontline, 18, 21-24, (2001 27 April).

Nations for Mental Health. 1997. An overview of a strategy to improve the mental health of underserved populations. Geneva: World Health Organization.

Pan-African Study Group on Onchocercal Skin Disease. 1995. The Importance of Onchocercal Skin Disease: Report of a Multi-Country Study. Applied Field Research Reports, No. 1. Geneva: UNDP/World Bank/WHO Special Programme for Research and Training in Tropical Diseases (TDR).

Paul, B. D. 1955. Health, Culture, \& Community: Case Studies of Public Reactions to Health Programs. New York: Russell Sage Foundation.

Raguram, R., Weiss, M. G., Keval, H., Channabasavanna, S. M. 2001. Cultural dimensions of clinical depression in Bangalore, India. Anthropology and Medicine, 8, ??-??. 
Raguram, R., Weiss, M. G., Channabasavanna, S. M., Devins, G. M. 1996. Stigma, depression, and somatization: A report from South India. American Journal of Psychiatry, 153, 1043-1049.

Rogler, L. H. 1989. The meaning of culturally sensitive research in mental health. Am J Psychiatry, 146, 296-303.

Romanucci-Ross, L. 1969. The hierarchy of resort in curtive processes: The Admiralty Islands, Melanesia. Journal of Health and Social Behavior, 10, 201-209.

Sartorius, N. 1973. Culture and the epidemiology of depression. Psychiatrica Neurologia Neurochirugia, 76, 479-487.

Schneider, Thomas. 2001. Conquering Europe, word for word. Op-Ed article. New York Times, 1 May 2001.

Shweder, R. 1999. Why cultural psychology? Ethos, 27, 62-73.

Sirey, J. A., Bruce, M. L., Alexopoulos, G. S., Perlick, D. A., Raue, P., Friedman, S. J., Meyers, B. S. 2001. Perceived stigma as a predictor of treatment discontinuation in young and older outpatients with depression. Am J Psychiatry, 158, 479-481.

Sperber, D. 1994. The modularity of thought and the epidemiology of representations. In: Hirschfeld, L. A., Gelman, S. A. (Eds). Mapping the Mind: Domain Specificity in Cognition and Culture. New York: Cambridge University Press, Pp. 39-67.

Sperber, D. 1990. The Epidemiology of Beliefs. In: Fraser, C., Gaskell, G. (Eds.). The Social Psychological Study of Widespread Beliefs. New York: Oxford University Press, Pp. $25-44$.

Sperber, D. 1985. Anthropology and psychology: towards an epidemiology of representations. Man, 29, 73-89.

Susser, M., Wtson, W., Hopper, K. 1985. Sociology in Medicine. Third Edition. New York: Oxford University Press. 
Uplekar, M., Rangan, S., Weiss, M. G., Ogden, J., Borgdorff, M. W., Hudelson, P. 2001. Attention to gender research in tuberculosis control. Int J Tub Lung Dis, 5, 220-224.

Vlassoff, C., Weiss, M. G., Ovuga, E. B. L., Eneanya, C., Nwel, P. T., Babalola, S. S., Awedoba, A. K., Theophilus, B., Cofie, P., and Shetabi, P. 2000. Gender and the stigma of onchocercal skin disease in Africa. Social Science and Medicine, 50, $1353-1368$.

Wellin, E. 1955. Water boiling in a Peruvian town. In: Paul, B. D. (Ed.), Health Culture and Community: Case Studies of Public Reactions to Health Programs. New York: Russell Sage Foundation; Chapter 3, Pp 71-103.

Weiss, M. G., Isaac, M., Parkar, S. R., Chowdhury, A. N. 2001a. Global, national, and local approaches to mental health: examples from India. Tropical Medicine and International Health, 6, 4-23.

Weiss, M. G., Jadhav, S., Raguram, R., Vounatsou, P., Littlewood, R. 2001b. Psychiatric stigma across cultures: local validation in Bangalore and London. Anthropology and Medicine, 8, ??-??.

Weiss, M. G. 2001. Psychiatric diagnosis and illness experience. In: Yilmaz, A.T., Weiss, M. G., and Riecher-Rössler, A. (eds), Cultural Psychiatry: Euro-International Perspectives. Basel: Karger Press. Bibliographica Psychiatrica Series, No. 169, Pp 2134.

Weiss, M. G. 1997. Explanatory model interview catalogue (EMIC): framework for comparative study of illness. Transcultural Psychiatry, 34, 235-263.

Weiss, M. G., Doongaji, D. R., Siddhartha, S., Wypij, D., Pathare, S., Bhatawdekar, M., Bhave, A., Sheth, A., Fernandes, R. 1992. The explanatory model interview catalogue (EMIC): contribution to cross-cultural research methods from a study of leprosy and mental health. British Journal of Psychiatry, 160, 819-830. 
World Bank. 2000. World Development Report 1999/2000. Entering the $21^{\text {st }}$ Century: The Changing Development Landscape. Oxford University Press. New York.

World Health Organization (WHO). 1983. Depressive Disorders in Different Cultures: Report on the Who Collaborative Study on Standardized Assessment of Depressive Disorders. Geneva: World Health Organization.

WHO. 1973. International Pilot Study of Schizophrenia: Report. Geneva: World Health Organization.

Yamamoto, J., Takeuchi, D. T., Sue, S., and Kurasaki, K. 1998. Cross-cultural epidemiology. Psychiatry and Clinical Neurosciences. 52(Suppl), S265-267.

Zielhuis, G. A., Kiemeney, L. A. A. L. M. 2001. Social epidemiology? No way. International Journal of Epidemiology, 30, 43-44. 
$-41-$

\section{Figures}

Figure 1. Complementary epidemiologies that inform clinical assessment

Figure 2. Framework for cultural epidemiology of illness representations

Figure 3. Approach to analysis of EMIC interview data 
Figure 1. Complementary epidemiologies that inform clinical assessment

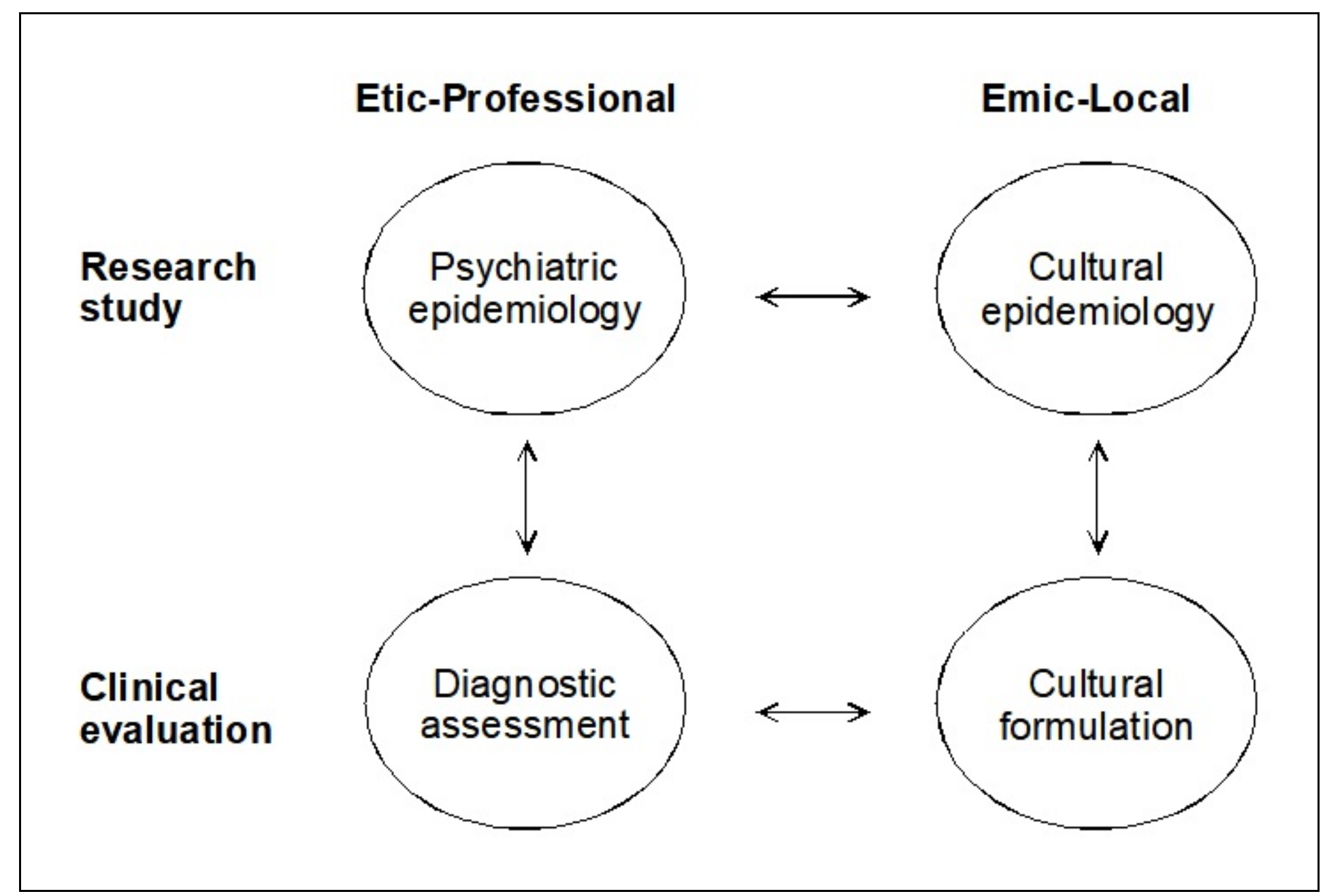


Figure 2. Framework for cultural epidemiology of illness representations

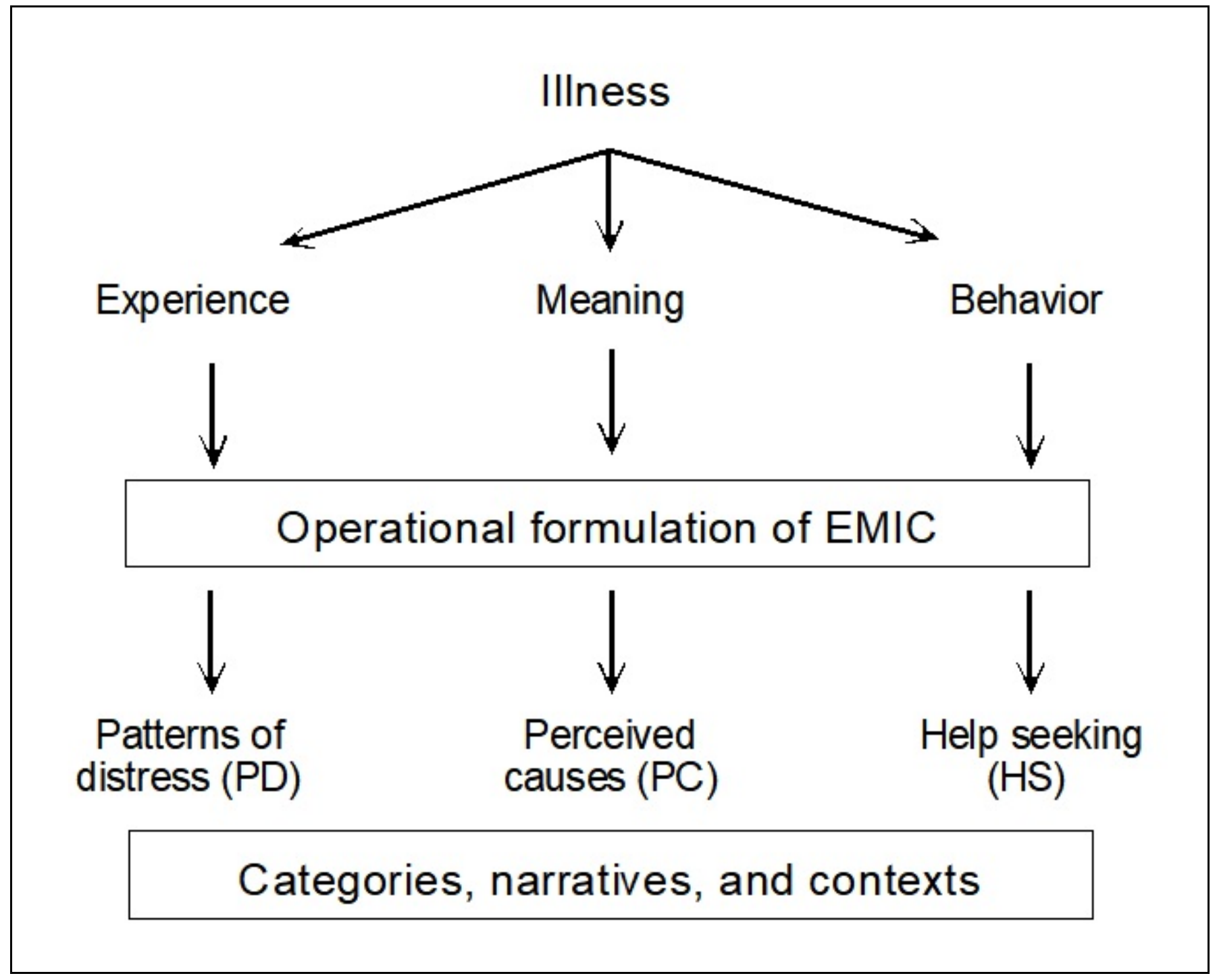


Figure 3. Approach to analysis of EMIC interview data

\section{Data set from EMIC Interviews}

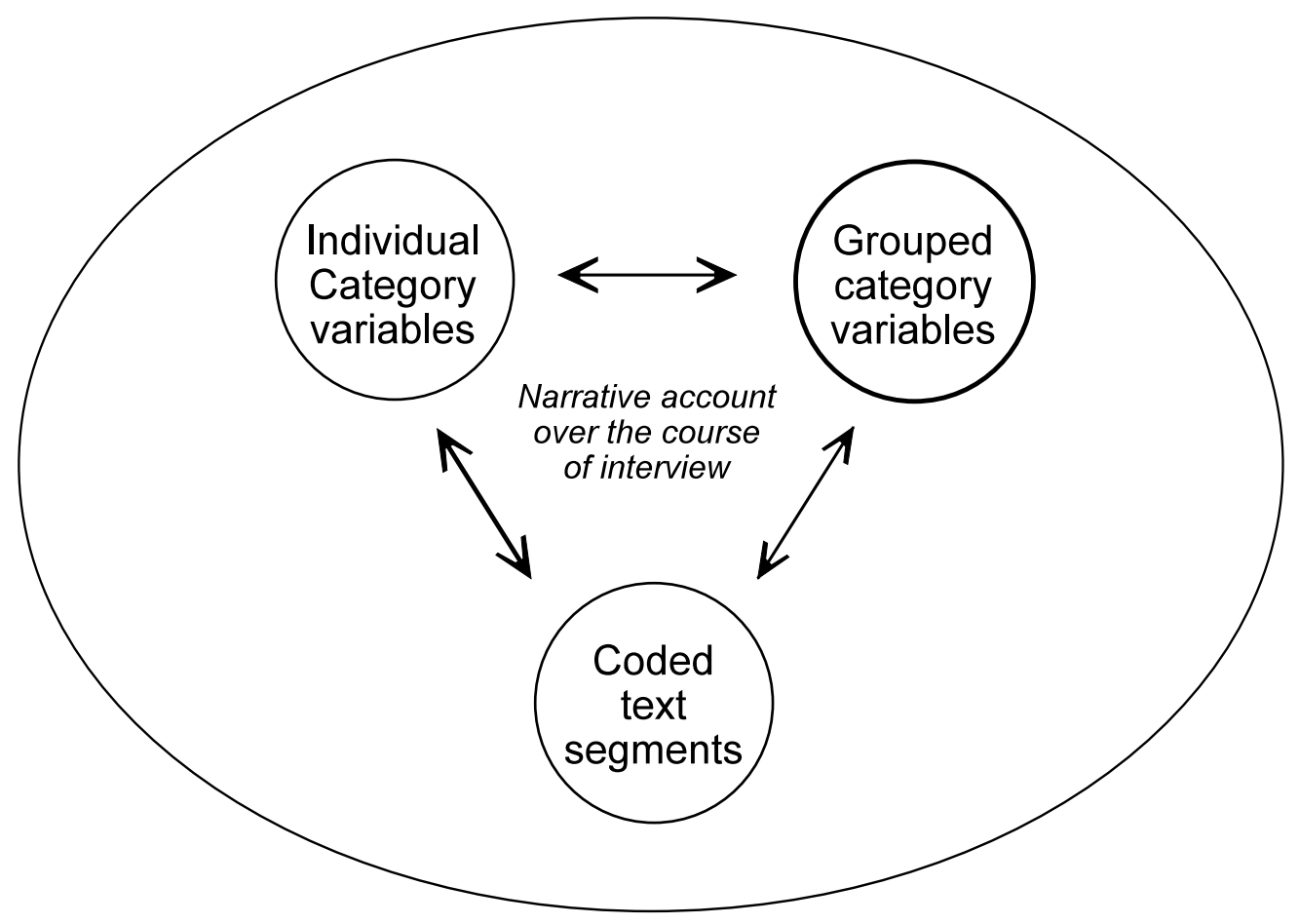

\section{Quantitative}

- Descriptive account (frequencies, etc.)

- Scales (eg, stigma) and latent variables (eg, prominence)

- Complementary data from clinical study and other sources

- Bivariate and multivariate relationships

\section{Qualitative}

- Descriptive account of categories (selected coded text segments)

- Comparison of narrative and coded segments among subgroups and across data sets

- Clarification of statistical relationships (coded text segments from selected respondents) 